IJMMS 30:1 (2002) 15-23

PII. S0161171202011560

http://ijmms.hindawi.com

(c) Hindawi Publishing Corp.

\title{
ON THE GEOMETRY OF FREE LOOP SPACES
}

\section{P. MANOHARAN}

Received 22 January 2001 and in revised form 18 June 2001

\begin{abstract}
We verify the following three basic results on the free loop space $L M$. (1) We show that the set of all points, where the fundamental form on $L M$ is nondegenerate, is an open subset. (2) The connections of a Fréchet bundle over $L M$ can be extended to $S^{1}$-central extensions and, in particular, there exist natural connections on the string structures. (3) The notion of Christoffel symbols and the curvature are introduced on $L M$ and they are described in terms of Christoffel symbols of $M$.
\end{abstract}

2000 Mathematics Subject Classification: 53Cxx, 58Dxx.

1. Introduction. In this paper, we consider the infinite-dimensional Fréchet manifold $L M$, the free loop space on $M$, which is the space of all smooth maps from the circle $S^{1}$ to a finite-dimensional manifold $M$. We discuss three different topics as a contribution to the general knowledge on the geometry of loop spaces.

If $M$ is a finite-dimensional Riemannian manifold, Atiyah [1] indicates that $L M$ has a fundamental closed 2-form $\omega$ which, unlike the finite-dimensional case, can be degenerate at certain points. A point in $L M$ is a smooth map $\phi: S^{1} \rightarrow M$. The Levi-Civita connection on $M$ induces a connection on the pullback bundle $\phi^{*} T M$, and hence a covariant operator $D_{\phi}$. The fundamental form $\omega$ is degenerate, precisely at those $\phi$ for which $D_{\phi}$ has zero eigenvalue. In the first part of this paper, by using the Nash embedding theorem [4], we show that the set of points, where $\omega$ is nondegenerate, is an open set.

Loop spaces are of a particular interest to physicists working on the grand unification theory. String theory involves a theory of spinors on $L M$; a string structure is defined as a lifting of the structural group to an $S^{1}$-central extension of the loop group [3]. Let $G \rightarrow P \rightarrow X$ be a principal Fréchet bundle over a Fréchet manifold $X$ that has enough smooth functions to admit a smooth partition of unity. Let $S^{1} \rightarrow \widetilde{G} \rightarrow G$ be an $S^{1}$-central extension of $G$ and let $\widetilde{G} \rightarrow \widetilde{P} \rightarrow X$ be a lifting of the principal bundle $G \rightarrow P \rightarrow X$. Although the existence of connections on a general Fréchet bundle is in general not guaranteed, the second part of this paper verifies that every connection on the principal bundle $G \rightarrow P \rightarrow X$ together with a $\widetilde{G}$-invariant connection on $S^{1} \rightarrow \widetilde{P} \rightarrow P$ yields a connection on $\widetilde{G} \rightarrow \widetilde{P} \rightarrow X$. In particular, as a corollary of this result, we prove that there exist connections on the string structures of $L M$.

In the last part of this paper, we give a detailed construction of Christoffel symbols on $L M$ by using "Fourier coordinates" and compute the corresponding curvature. Both Christoffel symbols and the curvature on $L M$ are given in terms of the Christoffel symbols on $M$ by a Fourier type series. We hope that these constructions of Christoffel 
symbols and the curvature will be useful to obtain local geometrical results on $L M$. The proofs of our results are rather straightforward and do not use any sophisticated method of functional analysis or differential geometry.

2. Fundamental form. The tangent space $T_{\phi}(L M)$ at any $\phi \in L M$ can be identified with $\Gamma\left(\phi^{*} T M\right)$, the sections of the pullback bundle $\phi^{*} T M \rightarrow S^{1}$ of the tangent bundle $T M \rightarrow M$. For any fixed nonnegative integer $r$, we have an inner product $\langle\langle,\rangle\rangle_{r}: \Gamma\left(\phi^{*} T M\right) \times \Gamma\left(\phi^{*} T M\right) \rightarrow R$ defined by

$$
\left\langle\left\langle s, s^{\prime}\right\rangle\right\rangle_{r}=\sum_{i=0}^{r} \int_{S^{1}}\left\langle D^{i} s(t), D^{i} s^{\prime}(t)\right\rangle d t,
$$

where $D$ is the covariant derivative of the Riemannian connection on $M$. This can be viewed as a Riemannian structure on $L M$ for each $r \geq 0$. Define $\omega_{\phi}^{(r)}: T_{\phi}(L M) \times$ $T_{\phi}(L M) \rightarrow R$ by $\omega_{\phi}^{(r)}(\alpha, \beta)=\langle\langle D \alpha, \beta\rangle\rangle_{r}$ and we can easily see that the fundamental form $\omega_{\phi}^{(r)}$ is bilinear and skew-symmetric. The energy function is defined by $e^{(r)}(\phi)=$ $\langle\langle D \phi, D \phi\rangle\rangle_{r}$.

REMARK 2.1. Hereafter, we simply use the notations $\omega$ and $e$ for $\omega^{(r)}$ and $e^{(r)}$, respectively.

We verify that (i) $\omega$ is a closed form and (ii) $d e+i_{A} \omega=0$, where $i_{A}$ is the contraction along the vector field $A$ associated to the natural $S^{1}$-action. Since $M$ can be isometrically embedded in $R^{N}$ for some large $N$ (by the Nash embedding theorem [4]), LM equipped with the Riemannian metric provided by $\langle\langle,\rangle\rangle_{r}$ is isometrically embedded in $L R^{N}$ with new metric provided by $\langle\langle,\rangle\rangle_{r}$ for each $r \geq 0$. Therefore, it is enough to prove (i) and (ii) for $M=R^{N}$. In this case, the vector field $A$ on an open subset $U$ of $L R^{N}$ is given by $(A \boldsymbol{\phi})(t)=\dot{\boldsymbol{\phi}}(t)$. Define the 1 -form $\theta: U \times L R^{N} \rightarrow R$ as the following composition:

$$
U \times L R^{N} \stackrel{A \times \text { id }}{\longrightarrow} L R^{N} \times L R^{N} \stackrel{\langle\langle,\rangle\rangle_{r}}{\longrightarrow} R
$$

so that $\theta(\phi, \alpha)=\langle\langle D \phi, \alpha\rangle\rangle_{r}$. Clearly $\theta$ is a smooth 1 -form and $d \theta=2 \omega$. Therefore, $\omega$ is a smooth closed form. Now

$$
\begin{aligned}
d e(\phi, \alpha) & =\lim _{s \rightarrow 0} \frac{1}{s}[e(\phi+s \alpha)-e(\phi)] \\
& =\lim _{s \rightarrow 0} \frac{1}{s}\left[\langle\langle D \phi+s D \alpha, D \phi+s D \alpha\rangle\rangle_{r}-\langle\langle D \phi, D \phi\rangle\rangle_{r}\right] \\
& =2\langle\langle D \phi, D \alpha\rangle\rangle_{r}=-2\left\langle\left\langle D^{2} \phi, \alpha\right\rangle\right\rangle_{r} \\
& =-2 \omega_{\phi}(A(\phi), \alpha)=-\left(i_{A} \omega\right)(\phi, \alpha)
\end{aligned}
$$

Hence $d e+i_{A} \omega=0$. The fundamental 2-form $\omega$ described above can be degenerate by the following lemma.

LEMMA 2.2. The fundamental form $\omega$ is degenerate at $\phi$ if and only if the corresponding covariant derivative $D_{\phi}$ has zero eigenvalue. 
Proof. The fundamental form $\omega$ is degenerate at $\phi$

$$
\begin{aligned}
& \Longleftrightarrow \exists \alpha \in T_{\phi}(L M) \quad \text { such that } \omega_{\phi}(\alpha, \beta)=0 \forall \beta \in T_{\phi}(L M) \\
& \Longleftrightarrow\left\langle\left\langle D_{\phi} \alpha, \beta\right\rangle\right\rangle_{r}=0 \quad \forall \beta \\
& \Longleftrightarrow D_{\phi} \alpha=0 .
\end{aligned}
$$

In other words, $\omega$ has degeneracy at $\phi$ if and only if there exists $\alpha$ in $T_{\phi}(L M)$ which is parallel along $\phi$. For example, $\omega$ is degenerate at any closed geodesic, since $\dot{\boldsymbol{\phi}}$ is parallel along a closed geodesic $\phi$.

THEOREM 2.3. The set of points where $\omega$ is nondegenerate is an open subset of LM.

Proof. For each $x \in M^{n}$ and $\tau \in L M$ that passes through $x$, the parallel transport $H_{x}(\tau)$ of $T_{x}(M)$ along $\tau$ is an element of the holonomy group $H_{x}$ of $M$ at $x$. Since the length of a vector and the angle between two vectors are preserved by the parallel transports of the Levi-Civita connection along a curve, the holonomy group at each point $x$ of a connected orientable manifold $M$ is a subgroup of $\mathrm{SO}(n)$. The fundamental form $\omega$ is degenerate at some $\phi$ if and only if for some $\theta$ (and hence for any $\theta$ ) the corresponding element of the holonomy group $H_{\phi(\theta)}$ has eigenvalue 1 . Notice that every element of $\mathrm{SO}(n)$ has eigenvalue 1 if $n$ is odd and hence in this case $\omega$ is degenerate at every $\phi \in L M$.

So we should restrict our attention to only the even-dimensional manifolds. For every $A \in \mathrm{SO}(n)$, let $e v_{1}(A)$ be the evaluation of the characteristic polynomial of $A$ at 1 . Let $\lambda$ be the following composition:

$$
\begin{aligned}
L M \stackrel{i_{\theta}}{\longrightarrow} L M \times S^{1} \stackrel{H}{\rightarrow} \mathrm{SO}(n) \stackrel{e v_{1}}{\longrightarrow} R, \\
\phi \longmapsto(\phi, \theta) \longmapsto H_{\phi(\theta)}(\phi) .
\end{aligned}
$$

We can easily see that $\lambda$ is independent of $\theta$ and $\omega$ is degenerate at $\phi$ if and only if $\lambda(\phi)=0$. Hence we have proved the theorem.

REMARK 2.4. Notice that whether the set of all nonsingular points of $\omega$ is dense or nondense in $L M$ heavily depends on the Levi-Civita connection of $M$. For example, if the curvature of $M$ is zero on a nonempty open subset of $M$, then the set of points, where $\omega$ is degenerate, contains a nonempty open subset of $L M$ and hence its complement, the set of points where $\omega$ is nondegenerate, is not dense in $L M$. This will not be the case if the Riemannian metric on $M$ (supposed to be a real analytic manifold) is real analytic. In this case, the set of degenerate points is a real analytic subset and hence of topological codimension 1 . Then the set of nondegenerate points is dense.

3. Lifting of connections. A Fréchet space $F$ is called nice if for every open subset $U$ of $F$ there exists a nonzero real-valued smooth function which vanishes outside $U$. For example, the space of sections of a smooth vector bundle over a compact connected finite-dimensional manifold is a nice Fréchet space and hence $L M$ is locally modeled on nice Fréchet spaces. A manifold locally modeled on nice Fréchet spaces has enough smooth functions to admit smooth partitions of unity. Hereafter, we assume that $X$ is a manifold locally modeled on nice Fréchet spaces. 
DEFINITION 3.1. A connection on a principal $G$-bundle $G \rightarrow P \stackrel{\pi}{\rightarrow} X$ is a smooth $G$-invariant splitting map $\mu: \pi^{*} T X \rightarrow T P$ in the exact sequence

$$
0 \longrightarrow T_{v} P \longrightarrow T P \stackrel{\mu}{\longrightarrow} \pi^{*} T X \longrightarrow 0
$$

of the bundles over $P$ where $T_{v} P$ is the vertical tangent bundle.

Let $S^{1} \rightarrow \widetilde{G} \rightarrow G$ be an $S^{1}$-central extension of the Fréchet Lie group $G$, or in other words, $S^{1}$ lies in the center of $\widetilde{G}$ and $\widetilde{G} / S^{1}=G$. Let $\widetilde{G} \rightarrow \widetilde{P} \stackrel{\tilde{\pi}}{\rightarrow} X$ be a lifting of $G \rightarrow P \rightarrow X$. Then we have the diagram

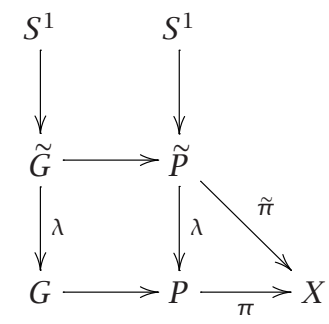

Of course, a connection on $G \rightarrow P \rightarrow X$ together with a connection on $S^{1} \rightarrow \widetilde{P} \rightarrow P$ may not yield a connection on $\widetilde{G} \rightarrow \widetilde{P} \rightarrow X$, because a splitting map $\lambda^{*} T P \stackrel{\mu}{\longrightarrow} T \widetilde{P}$ corresponding to a connection on $S^{1} \rightarrow \widetilde{P} \rightarrow P$ is $S^{1}$-invariant but not necessarily $\tilde{G}$-invariant.

Proposition 3.2. The space $\mathscr{C}_{\widetilde{G}}\left(S^{1} \rightarrow \widetilde{P} \rightarrow P\right)$ of $\widetilde{G}$-invariant connections is nonempty.

Proof. Let $T_{v}^{S^{1}} \widetilde{P}$ be the vertical tangent bundle over $\widetilde{P}$ corresponding to the bundle $S^{1} \rightarrow \widetilde{P} \rightarrow P$. We wish to show that we can produce a $\tilde{G}$-invariant splitting map $\mu$ in

$$
0 \longrightarrow T_{v}^{S^{1}} \widetilde{P} \longrightarrow T \widetilde{P} \stackrel{\mu}{\longrightarrow} \lambda^{*} T P \longrightarrow 0
$$

or equivalently a $\widetilde{G}$-invariant splitting map $\eta$ in

$$
0 \longrightarrow T_{v}^{S^{1}} \widetilde{P} \stackrel{\eta}{\longrightarrow} T \widetilde{P} \longrightarrow \lambda^{*} T P \longrightarrow 0 .
$$

Fortunately, $T_{v}^{S^{1}} \widetilde{P}$ is the trivial 1-dimensional bundle over $\widetilde{P}$. Indeed, for each $x \in \widetilde{P}$, consider the map $\{x\} \times S^{1} \rightarrow \widetilde{P}$ defined by $(x, \theta) \mapsto \theta \cdot x$. Since $S^{1}$ acts locally free, the derivative of this map, defined as

$$
\begin{aligned}
\{x\} \times T_{e}\left(S^{1}\right) & \longrightarrow\left(T_{v}^{S^{1}} \tilde{P}\right)_{x}, \\
(x, v) & \longmapsto v_{x},
\end{aligned}
$$

provides a nonzero vector field $A_{v}$ and hence $T_{v}^{S^{1}} \widetilde{P} \rightarrow \widetilde{P}$ is trivial.

Since $S^{1}$ lies in the center of $\widetilde{G}$, the action of $\widetilde{G}$ and that of $S^{1}$ commutes. Hence $A_{v}$ is $\widetilde{G}$-invariant and descends to a nonzero vector field on $T_{v}^{S^{1}} \widetilde{P} / \widetilde{G} \rightarrow \widetilde{P} / \widetilde{G}=X$. Thus 
$T_{v}^{S^{1}} \widetilde{P} / \widetilde{G} \rightarrow \widetilde{P} / \widetilde{G}$ is also a trivial line bundle. So it is enough to define a morphism (splitting map) $\gamma$ in

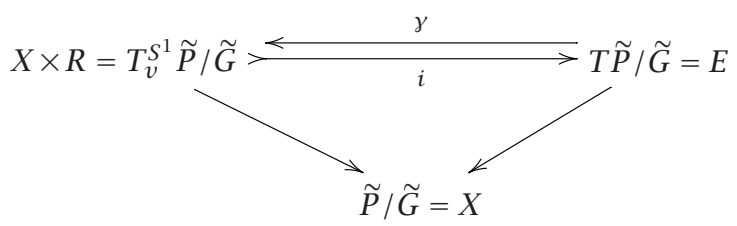

such that $\gamma \circ i=i d$. Since $X$ admits partitions of unity and the convex combination of splittings is a splitting, it is enough to produce a splitting locally on

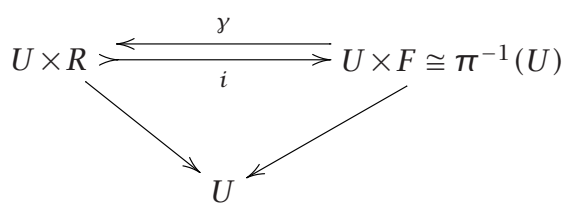

For each $x \in U$, let $v(x)=p_{2} i(x, 1) \in F$, where $p_{2}$ is the projection map. Choose a point $x_{0} \in U$. Since Hahn-Banach theorem holds on any Fréchet space $F$, there exists a linear map $\psi: F \rightarrow R$ such that $\psi\left(v\left(x_{0}\right)\right)=1$. By restricting to a smaller open subset $U_{1} \subseteq U$, we can assume that $\psi(v(x)) \neq 0$ for all $x \in U$. Let $\mathscr{L}(F, R)$ be the space of linear maps from $F$ to $R$. Define $\gamma: U \rightarrow \mathscr{L}(F, R)$ by

$$
\gamma(x)(f)=\frac{\psi(f)}{\psi(v(x))} \quad \forall f \in F .
$$

Since $\gamma(x)(v(x))=1$ for all $x, \gamma$ yields a splitting map. This completes the proof of the lemma and immediately yields the following theorem.

THEOREM 3.3. Every connection on the principal Fréchet bundle $G \rightarrow P \rightarrow X$ together with a $\tilde{G}$-invariant connection on $S^{1} \rightarrow \widetilde{P} \rightarrow P$ yields a connection on $\widetilde{G} \rightarrow \widetilde{P} \rightarrow X$.

REMARK 3.4. The lifting of a $G$-connection on $\mathrm{P}$ to a $\widetilde{G}$-connection on $\widetilde{P}$ is not unique. The failure of uniqueness is measured by $\widetilde{G}$-invariant $S^{1}$-connections on $S^{1} \rightarrow$ $\widetilde{P} \rightarrow P$ and hence by a 1-dimensional form on $\widetilde{P}$ which is $\widetilde{G}$-invariant.

COROLLARY 3.5. If $F$ is any Fréchet space with a $\widetilde{G}$-action, then every connection on $G \rightarrow P \rightarrow X$ defines at least one connection on $\widetilde{P} \times_{\tilde{G}} F \rightarrow X$.

Proof. The proof is the same as the finite-dimensional case. Every connection on $\widetilde{P}$ defines a connection on $\widetilde{P} \times F \rightarrow X$. Since $\widetilde{P} \times F \rightarrow \widetilde{P} \times_{\widetilde{G}} F$ is a submersion and the horizontal subspaces are mapped injectively (or in other words, horizontal subspaces of $\widetilde{P} \times F$ intersect trivially with the kernel), there is an induced connection on $\widetilde{P} \times_{\widetilde{G}} F \rightarrow X$.

Let $M^{n}$ be an even-dimensional smooth compact connected orientable manifold with the spin structure $\operatorname{Spin}(n) \rightarrow Q \rightarrow M$. Let $L \operatorname{Spin}(n) \rightarrow L Q \rightarrow L M$ be the associated $L \operatorname{Spin}(n)$-principal bundle. It is shown in [3] that such a bundle can be lifted to a 
new bundle $\widetilde{L \operatorname{Spin}(n)} \rightarrow \widetilde{L Q} \rightarrow L M$ provided that $p_{1}(M)=0$, where $p_{1}(M)$ is the first Pontrjagin class of $M$ and $L \widetilde{\operatorname{Spin}(n)}$ is the nontrivial $S^{1}$-central extension of $L \operatorname{Spin}(n)$. The spinors on $L M$ are defined as the sections of the vector bundles $\widetilde{L Q} \times \underset{L \operatorname{Spin}(n)}{ } \Lambda^{ \pm} \rightarrow$ $L M$ where $L \widetilde{\operatorname{Spin}(n)}$ acts on $\Lambda^{ \pm}$by two inequivalent irreducible representations. The reader may consult [5] for the construction of such infinite-dimensional irreducible representations.

COROLLARY 3.6. Any connection on $L \operatorname{Spin}(n) \rightarrow L Q \rightarrow L M$ induces connections on the spin bundles $\widetilde{L Q} \times \underset{L \operatorname{Spin}(n)}{\widetilde{L}} \Lambda^{ \pm} \rightarrow L M$.

4. Christoffel symbols on $L M$. If $M^{n}$ is an orientable Riemannian manifold and $\phi \in L M$, we can choose a frame $\left\{e_{1}, \ldots, e_{n}\right\}$ of the pullback bundle $\phi^{*} T M \rightarrow S^{1}$. Consider the Riemannian structures $\langle\langle,\rangle\rangle_{r, \phi}$ induced by the Riemannian structure of $M$. Let $s_{1}, s_{2} \in T_{\phi}(L M)$ with $s_{1}=\sum s_{1}^{i} e_{i}$ and $s_{2}=\sum s_{2}^{j} e_{j}$. We can explicitly calculate $\left\langle\left\langle s_{1}, s_{2}\right\rangle\right\rangle_{r, \phi}$. For example, if $r=0$,

$$
\begin{aligned}
\left\langle\left\langle s_{1}, s_{2}\right\rangle\right\rangle_{0, \phi} & =\int_{S^{1}}\left\langle s_{1}, s_{2}\right\rangle(\theta) d \theta \\
& =\sum_{i, j=1}^{n} \int_{S^{1}} s_{1}^{i}(\theta) s_{2}^{j}(\theta) g_{i j}(\phi(\theta)) d \theta,
\end{aligned}
$$

where $g_{i j}(\phi(\theta))=\left\langle e_{i}(\phi(\theta)), e_{j}(\phi(\theta))\right\rangle$; and if $r=1$,

$$
\begin{aligned}
\left\langle\left\langle s_{1}, s_{2}\right\rangle\right\rangle_{1, \phi}= & \sum \int_{S^{1}} s_{1}^{i}(\theta) s_{2}^{j}(\theta) g_{i j}(\phi(\theta)) d \theta \\
& +\sum \int_{S^{1}} \dot{\phi}\left(s_{1}^{i}\right)(\theta) \dot{\boldsymbol{\phi}}\left(s_{2}^{j}\right)(\theta) g_{i j}(\phi(\theta)) d \theta \\
& +\sum \int_{S^{1}} \dot{\boldsymbol{\phi}}\left(s_{1}^{i}\right)(\theta) \dot{\phi}^{t}(\theta) s_{2}^{j}(\theta) \Gamma_{t j}^{k}(\phi(\theta)) g_{k i}(\phi(\theta)) d \theta \\
& +\sum \int_{S^{1}} \dot{\boldsymbol{\phi}}\left(s_{2}^{j}\right)(\theta) \dot{\phi}^{u}(\theta) s_{1}^{i}(\theta) \Gamma_{u i}^{l}(\phi(\theta)) g_{l j}(\phi(\theta)) d \theta \\
& +\sum \int_{S^{1}} \dot{\phi}^{t}(\theta) \dot{\phi}^{u}(\theta) s_{1}^{i}(\theta) s_{2}^{j}(\theta) \Gamma_{t i}^{k} \Gamma_{u j}^{l} g_{k l}(\phi(\theta)) d \theta,
\end{aligned}
$$

where $\left\{\Gamma_{i j}^{k}(x)\right\}$ represent the Christoffel symbols of the Levi-Civita connection on $M$. We use the Einstein convention where the sums run through super- and subscripts. In all of our further calculations, we restrict ourselves to the case $r=0$, and denote $\langle\langle,\rangle\rangle_{0, \phi}$ simply by $\langle\langle,\rangle\rangle_{\phi}$ for each $\phi \in L M$.

A connection on a Fréchet vector bundle $E \stackrel{\pi}{\rightarrow} X$ over a Fréchet manifold $X$ is a rule that assigns to each point of $E$ a complementary subspace for the vertical tangent space, called the horizontal subspace, such that the local representation of the connection is given by a smooth map $\Gamma$ as follows: if $\pi$ is locally $U \times G \rightarrow U$ where 
$U \subseteq F$ is open and $F$ and $G$ are Fréchet spaces (i.e., $X$ is locally modeled by $F$ and $G$ is the fiber), then the horizontal vectors consists of all $(b, c) \in F \times G$ with $c=\Gamma(u, a, b)$ where $\Gamma: U \times G \times F \rightarrow G$ is bilinear in $a$ and $b$ (see [2]).

By a connection on a manifold $X$, we always understand a connection on its tangent bundle. Unlike Banach manifolds, the existence of a connection on an arbitrary Fréchet manifold is not guaranteed. However, every connection $\Gamma$ on $M$ determines a connection on $L M$, also denoted by $\Gamma$, which is given locally at $\phi \in L M$ by the formula

$$
\Gamma(\phi, f, g)(\theta)=\Gamma(\phi(\theta), f(\theta), g(\theta)),
$$

where $f(\theta), g(\theta) \in T_{\phi(\theta)} M$ and $\Gamma$ is bilinear in the last two variables.

Every $f \in L R^{n}$ has Fourier series

$$
f \sim \sum_{p \in Z}\left(f_{1 p}, f_{2 p}, \ldots, f_{n p}\right) \alpha_{p}(\theta)
$$

such that $\sum_{p}\left\|\vec{f}_{p}\right\|^{2} p^{2 k}<\infty$ for every integer $k$, where

$$
\alpha_{p}(\theta)= \begin{cases}\cos p \theta & \text { if } p \leq 0 \\ \sin p \theta & \text { if } p>0\end{cases}
$$

In other words, $f \sim \sum_{p \in Z} \sum_{k=1}^{n} f_{k p} \alpha_{p}(\theta) \vec{e}_{k}$ where $\left\{\vec{e}_{1}, \ldots, \vec{e}_{n}\right\}$ is the standard basis of $R^{n}$. The numbers $\left\{f_{k p}\right\}$ are called the Fourier coordinates of $f \in L R^{n}$. Notice that though every element $f \in L R^{n}$ has Fourier coordinates which is a sequence $\left\{f_{k p}\right\}$ of real numbers, only the sequences $\left\{f_{k p}\right\}$ satisfying additional convergence conditions are coordinates for elements of $L R^{n}$ (see [6, Proposition 10.2]).

By choosing a frame $\left\{e_{1}, \ldots, e_{n}\right\}$ of the pullback bundle $\phi^{*} T M \rightarrow S^{1}$, just like above (beginning of Section 4 ), each $s \in \Gamma\left(\phi^{*} T M\right)$ can be assigned Fourier coordinates $\left\{s_{k p}\right\}$ where $s \sim \sum_{p \in Z} \sum_{k=1}^{n} s_{k p} \alpha_{p}(\theta) e_{k}$. Let $\Gamma$ be a connection on $L M$. For a fixed $\phi \in L M$, let $\left\{E_{k p}(\phi)\right\}_{1 \leq k \leq n, p \in Z}$ be defined by $E_{k p}(\phi)(\theta)=\alpha_{p}(\theta) e_{k}(\phi(\theta))$. We define the Christoffel symbols $\left\{\widetilde{\Gamma}_{k p, l q, m r}(\phi)\right\}$ of $\Gamma$ with respect to $\left\{E_{k p}(\phi)\right\}$ by

$$
\widetilde{\Gamma}_{k p, l q, m r}(\phi)=\left\langle\left\langle\Gamma\left(\phi, E_{k p}, E_{l q}\right), E_{m r}\right\rangle\right\rangle_{\phi},
$$

where $1 \leq k, l, m \leq n$ and $p, q, r \in \mathbb{Z}$.

Note 1. Similar definition in the finite-dimensional case yields $\left\{\widetilde{\Gamma}_{\beta \gamma \delta}(x)\right\}$ which are connected to the usual Christoffel symbols $\left\{\Gamma_{\beta \gamma}^{\delta}(x)\right\}$ by

$$
\Gamma_{\beta \gamma}^{\delta}(x)=\widetilde{\Gamma}_{\beta \gamma \mu}(x)\left(g^{-1}(x)\right)^{\delta \mu} .
$$

Now we can describe the Christoffel symbol $\widetilde{\Gamma}$ in terms of the Christoffel symbols of $M$ as follows.

Proposition 4.1. Let $\left\{\Gamma_{k l}^{m}\right\}$ be the Christoffel symbols with respect to a connection $\Gamma$ of $M$ and $\left\{\widetilde{\Gamma}_{k p, l q, m r}\right\}$ be the Christoffel symbols of the induced connection $\Gamma$ of $L M$. Then

$$
\widetilde{\Gamma}_{k p, l q, m r}(\phi)=\sum_{i=1}^{n} \int_{S^{1}} \alpha_{p}(\theta) \alpha_{q}(\theta) \alpha_{r}(\theta) \Gamma_{k l}^{i}(\phi(\theta)) g_{i m}(\phi(\theta)) d \theta
$$


Proof. The formula for Christoffel symbols can be obtained as follows:

$$
\begin{aligned}
& \left\langle\left\langle\Gamma\left(\phi, E_{k p}, E_{l q}\right), E_{m r}\right\rangle\right\rangle_{\phi} \\
& \quad=\int_{S^{1}}\left\langle\Gamma\left(\phi(\theta), E_{k p}(\phi)(\theta), E_{l q}(\phi)(\theta)\right), E_{m r}(\phi)(\theta)\right\rangle d \theta \\
& \quad=\int_{S^{1}} \alpha_{p}(\theta) \alpha_{q}(\theta) \alpha_{r}(\theta)\left\langle\Gamma\left(\phi(\theta), e_{k}(\phi(\theta)), e_{l}(\phi(\theta))\right), e_{m}(\phi(\theta))\right\rangle d \theta \\
& =\int_{S^{1}} \alpha_{p}(\theta) \alpha_{q}(\theta) \alpha_{r}(\theta) \tilde{\Gamma}_{k l m}(\phi(\theta)) d \theta \\
& =\sum_{i=1}^{n} \int_{S^{1}} \alpha_{p}(\theta) \alpha_{q}(\theta) \alpha_{r}(\theta) \Gamma_{k l}^{i}(\phi(\theta)) g_{i m}(\phi(\theta)) d \theta .
\end{aligned}
$$

This completes the proof of the proposition.

The curvature $R$ of a connection $\Gamma$ is given locally by the map

$$
\begin{aligned}
R(\phi, f, g, h)= & D_{\phi} \Gamma\{f, g, h\}-D_{\phi} \Gamma\{f, h, g\} \\
& -\Gamma(\phi, \Gamma(\phi, f, g), h)+\Gamma(\phi, \Gamma(\phi, f, h), g)
\end{aligned}
$$

which is trilinear in the last three variables. Here $D_{\phi} \Gamma\{f, g, h\}$ is the derivative of $\Gamma$ at $(\phi, f, g)$ in the direction of $h$.

If $\Gamma$ (with the curvature $R$ ) is induced by the connection $\Gamma_{M}$ (with the curvature $R_{M}$ ) then

$$
R(\phi, f, g, h)(\theta)=R_{M}(\phi(\theta), f(\theta), g(\theta), h(\theta))
$$

for all $\theta \in S^{1}$. As before let

$$
\widetilde{R}_{k p, l q, m r, n s}(\phi)=\left\langle\left\langle R\left(\phi, E_{k p}, E_{l q}, E_{m r}\right), E_{n s}\right\rangle\right\rangle_{\phi} .
$$

Proposition 4.2. By a similar argument as in Proposition 4.1, we have the following formula for the curvature

$$
\widetilde{R}_{k p, l q, m r, n s}(\phi)=\sum_{i=1}^{n} \int_{S^{1}} \alpha_{p}(\theta) \alpha_{q}(\theta) \alpha_{r}(\theta) \alpha_{s}(\theta) R_{k l m}^{i}(\phi(\theta)) g_{i r}(\phi(\theta)) d \theta,
$$

where $R_{k l m}^{i}(x)$ is defined by

$$
R_{M}\left(x, e_{k}(x), e_{l}(x), e_{m}(x)\right)=R_{k l m}^{i}(x) e_{i}(x)
$$

for $x=\phi(\theta) \in M$.

\section{REFERENCES}

[1] M. F. Atiyah, Circular symmetry and stationary-phase approximation, Astérisque (1985), no. 131, 43-59.

[2] R. S. Hamilton, The inverse function theorem of Nash and Moser, Bull. Amer. Math. Soc. (N.S.) 7 (1982), no. 1, 65-222.

[3] D. A. McLaughlin, Orientation and string structures on loop space, Pacific J. Math. 155 (1992), no. 1, 143-156. 
[4] J. Nash, The imbedding problem for Riemannian manifolds, Ann. of Math. (2) 63 (1956), 20-63.

[5] A. Pressley and G. Segal, Loop Groups, Oxford Mathematical Monographs, Oxford University Press, New York, 1986.

[6] M. A. Shubin, Pseudodifferential Operators and Spectral Theory, 2nd ed., Springer-Verlag, Berlin, 2001.

P. MANOHARAN: MATHematics Department, UniVersity of MARYLAND, UNIVERSity COLLEGE, 3501 UNIVERSITY BOULEVARD EAST, ADELPHI, MD 20783, USA

E-mail address: pmanoharan@umuc. edu 


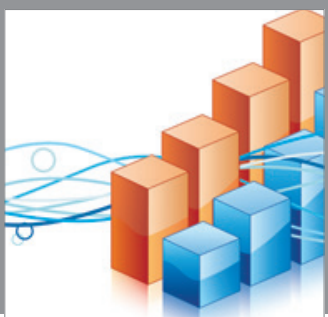

Advances in

Operations Research

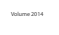

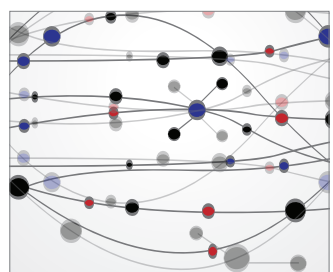

\section{The Scientific} World Journal
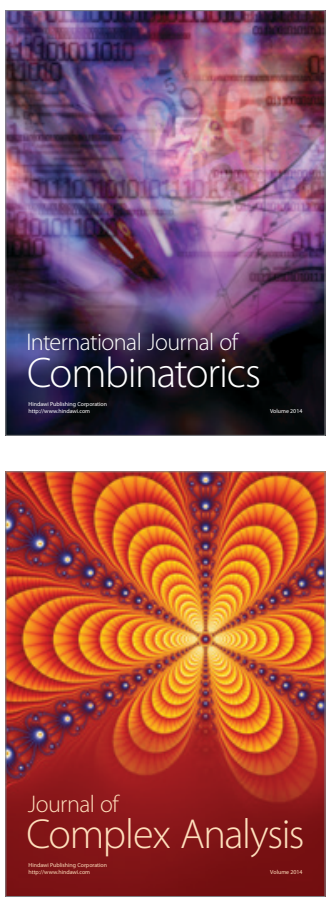

International Journal of

Mathematics and

Mathematical

Sciences
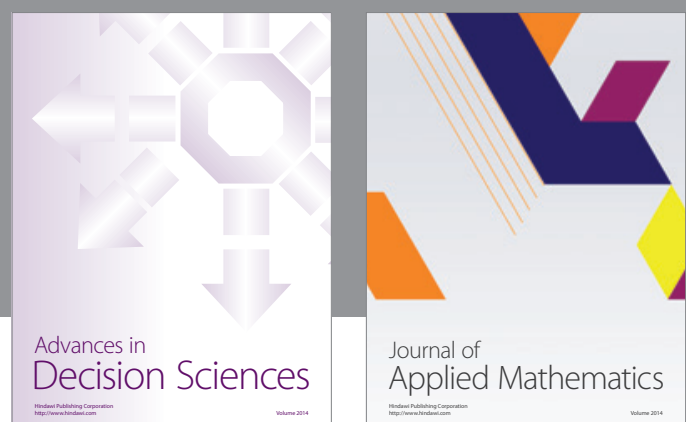

Journal of

Applied Mathematics
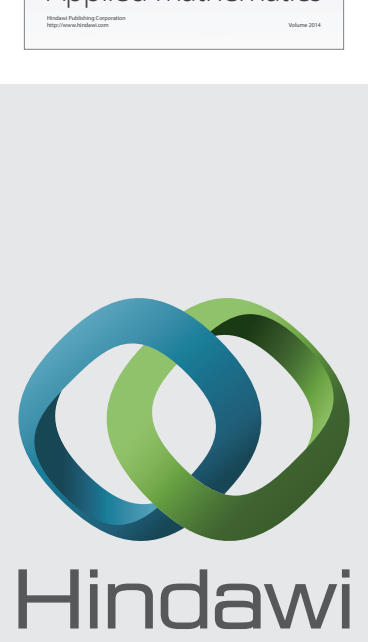

Submit your manuscripts at http://www.hindawi.com
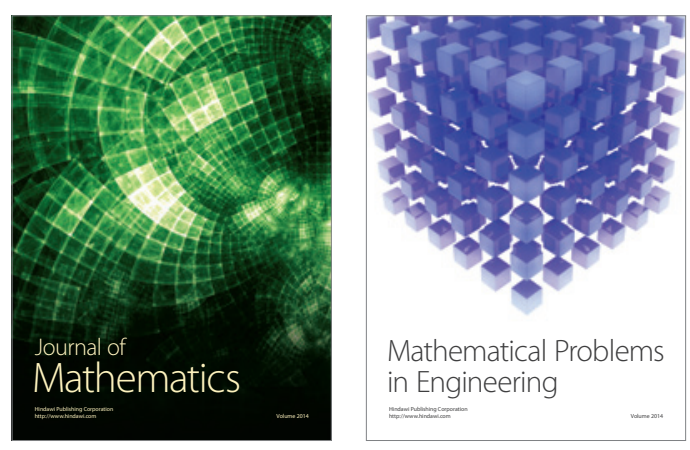

Mathematical Problems in Engineering
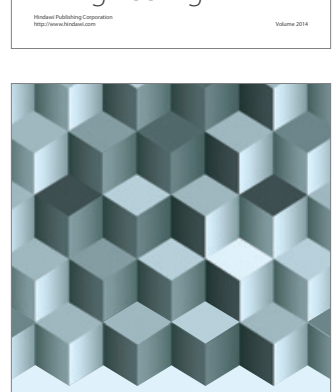

Journal of

Function Spaces
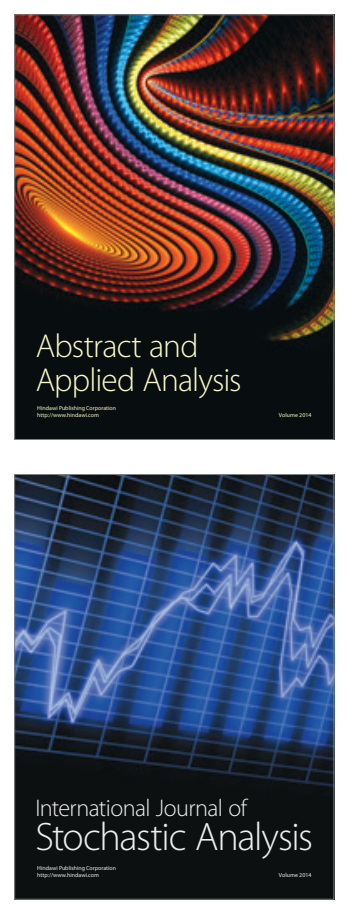

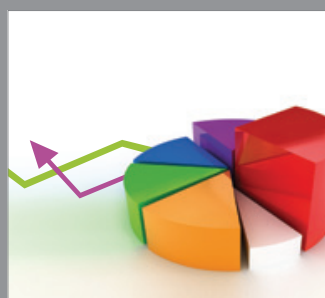

ournal of

Probability and Statistics

Promensencen
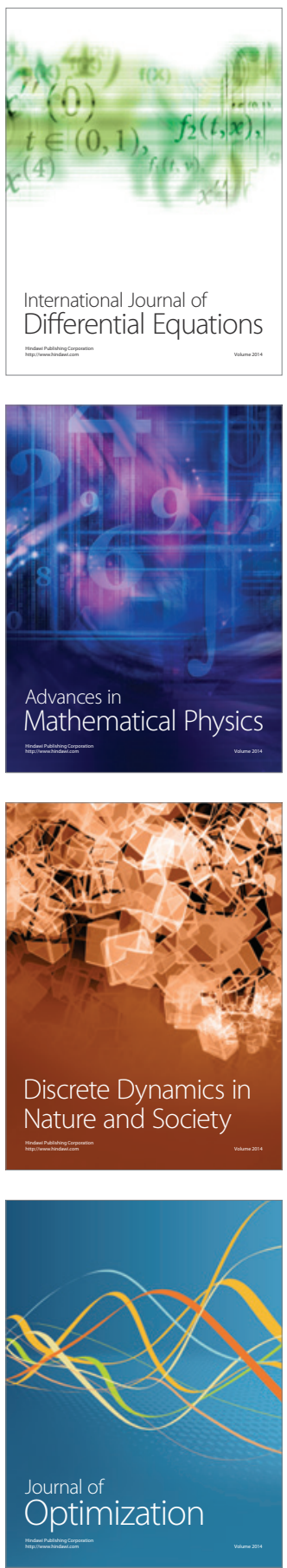